\title{
PARTICIPAÇÃO E CONTROLE SOCIAL NO CONTEXTO POLÍTICO DOS CUIDADOS PALIATIVOS NO BRASIL: UMA REFLEXÃO TEÓRICA
}

\section{PARTICIPATION AND SOCIAL CONTROL IN THE POLITIC CONTEXT OF PALLIATIVE CARE: A THEORETICAL REFLECTION}

\section{PARTICIPACIÓN Y CONTROL SOCIAL EN EL CONTEXTO POLÍTICO DE LOS CUIDADOS PALIATIVOS: UNA REFLEXIÓN TEÓRICA}

Jacqueline Resende Boaventura ${ }^{1}$, Juliana Dias Reis Pessalacia ${ }^{2}$, Tatiana Carvalho Reis Martins ${ }^{3}$, Alexandre Ernesto da Silva ${ }^{4}$, Maristela Rodrigues Marinho ${ }^{5}$, Priscila Kelly da Silva Neto 6.

\section{RESUMO}

Objetivo: Refletir acerca da efetivação do princípio da participação e controle social no contexto dos cuidados paliativos. Métodos: Reflexão teórica, fundamentada no princípio organizativo da 'participação popular', à luz da Lei n 8142 de 1990, que dispõe sobre a atuação da comunidade na gestão do Sistema Único de Saúde (SUS) brasileiro e em publicações nacionais e internacionais na temática. Resultados: Experiências referentes à presença popular, na formulação e gestão de políticas de saúde, para os cuidados paliativos nos países, são escassas. O envolvimento social em cuidados paliativos ocorre de forma limitada, em diferentes contextos, contemplando-se condições específicas, tais como avaliação da qualidade dos serviços, acesso a fármacos e decisões envolvendo terminalidade. No Brasil, a inserção dos cuidados paliativos, nas políticas públicas de saúde, é recente, porém deve contemplar os princípios estabelecidos pelo SUS. Acredita-se que o envolvimento popular proporcione ao paciente em cuidados paliativos e seus familiares voz ativa e autonomia e maior controle nas políticas públicas. Conclusão: Torna-se relevante o aprofundamento da discussão acerca da participação e controle social voltados a pacientes em CP e seus familiares, no âmbito da formulação e gestão de políticas públicas, para esses cuidados no Brasil e do papel da enfermagem no fortalecimento desse direito. Descritores: Cuidados paliativos. Participação do paciente. Controle social formal. Políticas de saúde. Enfermagem.

\begin{abstract}
Objective: To reflect on the implementation of the principle of participation and social control in the context of palliative care (PC). Methods: Theoretical reflection based on the organizational principle of 'popular participation', in the light of Law 8142 of 1990 , which provides for community participation in the management of the Brazilian Unified Health System and in national and international publications. Results: Experiences regarding popular participation in the formulation and management of health policies for PC in countries are scarce. Social involvement in PC occurs in a limited way in different contexts, considering specific conditions, such as quality of service assessment, access to drugs, and decisions involving terminality. In Brazil, the insertion of PC in public health policies is recent, but it should include the principles established in the unified health system. Popular involvement is believed to provide patients in PC and their families with active voice and autonomy, as well as greater control over public policies. Conclusion: It is relevant to deepen the discussion about participation and social control aimed at patients in PC and their families, in the context of the formulation and management of public policies for this care in Brazil and the role of nursing in strengthening this right. Descriptors: Palliative care; Patient Participation; Social control formal; Health policy; Nursing.
\end{abstract}

\section{RESUMEN}

Objetivo: Reflexionar sobre la implementación del principio de participación y control social en el contexto de los cuidados paliativos. Métodos: reflexión teórica, basada en el principio organizativo de 'participación popular', al amparo de la Ley $\mathrm{N}^{\circ} 8142$ de 1990, que prevé la participación comunitaria en la gestión del Sistema Único de Salud (SUS) de Brasil y en publicaciones nacionales e internacionales sobre esta temática. Resultados: las experiencias con respecto a la participación popular en la formulación y gestión de políticas de salud para cuidados paliativos en los países son escasas. La participación social en los cuidados paliativos se produce de forma limitada en diferentes contextos, considerando condiciones específicas, como la evaluación de la calidad del servicio, el acceso a los medicamentos y las decisiones que involucran la fase terminal. En Brasil, la inserción de los cuidados paliativos en las políticas de salud pública es reciente, pero debe incluir los principios establecidos en el SUS. Se cree que la participación popular brinda a los pacientes en cuidados paliativos y a sus familias, voz activa y autonomía, y un mayor control sobre las políticas públicas. Conclusión: es relevante profundizar la discusión sobre participación y control social dirigida a pacientes en PC y sus familias, en el contexto de la formulación y gestión de políticas públicas para esta atención en Brasil y el papel de la enfermería en el fortalecimiento de este derecho.

Descriptores: Cuidados paliativos; Participación del paciente; Control social formal; Políticas de salud; Enfermeria.

${ }^{1}$ Enfermeira, Especialista. Aluna do Programa de Pós-Graduação - Mestrado em Enfermagem UFMS. ${ }^{2}$ Enfermeira, Pós Doutora em Enfermagem. ${ }^{3}$ Enfermeira, Doutora em Ciências da Saúde. ${ }^{4}$ Enfermeiro, Doutor em Enfermagem. ${ }^{5}$ Enfermeria, Especialista pela Universidade Federal de Mato Grosso do Sul. ${ }^{6}$ Enfermeria, Especialista pela Universidade Federal de Mato Grosso do Sul. ; Available in: DOI: 


\section{INTRODUÇÃO}

Tradicionalmente, definem-se os Cuidados Paliativos (CP), segundo conceito proposto em 1990 e revisado em 2002 pela Organização Mundial de Saúde (OMS), como a "assistência promovida por uma equipe multidisciplinar, que objetiva a melhoria da qualidade de vida do paciente e seus familiares, diante de uma doença que ameace a vida, por meio da prevenção e alívio do sofrimento, da identificação precoce, avaliação impecável e tratamento de dor e demais sintomas físicos, sociais, psicológicos espirituais"(1).

Entretanto, recentemente, propôs-se, com base na reunião da Comissão Lancet de Acesso Global a Cuidados Paliativos e Alívio da Dor, em 2017, um conceito ainda mais amplo para esses cuidados. Define-se $\mathrm{CP}$, embasado no relatório dessa comissão, como cuidados holísticos e ativos a serem ofertados a pessoas de todas as idades e que vivenciam intenso sofrimento, proveniente de doença grave, em especial, aquelas que estão em fim de vida, tendo como objetivo a melhora na qualidade de vida de pacientes, familiares e cuidadores $^{(2)}$.

$\mathrm{O}$ cenário dos $\mathrm{CP}$ ainda apresenta-se em processo de estruturação política no Brasil, as estratégias de organização até então são desafiadoras, pois sua abordagem não contempla apenas a assistência ao paciente, mas, sim, um compromisso pelo cuidar e requer a integração e dedicação de uma equipe multidisciplinar ${ }^{(3)}$.

Assim, aprovou-se, em 2014, uma resolução, com a qual a Assembleia Mundial de Saúde (AMS) desafia todos os governos a inserirem os CP no planos e políticas de saúde. Além disso, delegou-se à Organização Mundial de Saúde (OMS) a responsabilidade de monitorização dessas ações ${ }^{(4)}$.

No Brasil, contudo tais cuidados desenvolvem-se de modo bastante frágil, de maneira isolada e dependentes quase sempre de doações, além de acesso limitado a medicações para o controle da dor. Evidencia-se, neste contexto, que pacientes com doenças graves convivem regularmente com a dor não controlada e não têm acesso a CP de qualidade no país ${ }^{(5)}$.

Tal problemática deve-se ao fato de que ainda não existe, no país, uma política de saúde pública específica, para a implementação e organização dos $\mathrm{CP}$, nos três níveis de complexidade do sistema de saúde. Assim, a organização atual dos CP baseia-se em portarias referentes à assistência ao paciente oncológico e na portaria Rede de Atenção à Saúde das Pessoas com Doenças Crônicas no âmbito do Sistema Único de Saúde (SUS) ${ }^{(5)}$.

Assim, alicerçada em várias discussões da Comissão Intergestores Tripartite (CIT) do Ministério da Saúde (MS) brasileiro, publicou-se, em novembro de 2018, no Diário Oficial da União (DOU), a Resolução $n^{\circ} 41$ que "Dispõe sobre as diretrizes, para a organização dos cuidados paliativos, à luz dos cuidados continuados integrados, no âmbito Sistema Único de Saúde (SUS) "(6). Essa resolução define em seu Art. 20 que: "Art. 2ㅇ Cuidados paliativos consistem na assistência promovida por uma equipe multidisciplinar, que objetiva a melhoria da qualidade de vida do paciente e seus familiares, diante de uma doença que ameace a vida, por meio da prevenção e alívio do sofrimento, da identificação precoce, avaliação impecável e tratamento de dor e demais sintomas físicos, sociais, psicológicos e espirituais"(6).

Destarte, a Resolução no 41 da CIT constitui-se uma referência a uma futura criação de uma política e portarias com estratégias que garantam a implementação do objetivo proposto na resolução ${ }^{(5)}$.

A participação popular, no contexto das políticas públicas de saúde, é um fundamento fortemente respeitado pelos governos e gestores em saúde. Agregar o paciente nas decisões, compreendendo seus valores e preferências, é primordial para a tomada de condutas na prática individual e formulação de diretrizes clínicas. No entanto há poucos estudos que abordam a melhor forma de compreender e obter uma percepção do usuário na formulação e atualização das políticas de saúde para os $\mathrm{CP}^{(7)}$.

Posto isto, considera-se necessária a busca de experiências na organização e envolvimento social na implementação de políticas públicas de saúde voltadas aos CP. Assim, busca-se refletir acerca da efetivação do princípio da participação e controle social no contexto dos CP no SUS brasileiro.

\section{MÉTODOS}

Trata-se de uma reflexão teórica, fundamentada no princípio da Participação Popular à luz da Lei $n^{\circ} 8142$ de 28 de dezembro de $1990^{(8)}$. O Sistema Único de Saúde (SUS) brasileiro prevê um sistema com princípios doutrinários e organizativos que é constantemente atualizado, por meio de leis, 
normas e regulamentações que vão sendo promulgadas, em função de adequações a novas necessidades da população. Os princípios normativos orientam a forma como o sistema deve funcionar, tendo como eixo norteador os princípios doutrinários ${ }^{(9)}$. A discussão foi embasada em artigos nacionais e internacionais que discorrem sobre a Participação Popular nas políticas de saúde referentes aos CP. Entretanto vale ressaltar que o conceito de Participação Popular, no Brasil, distingue-se do conceito de outros países. No Brasil, a Participação Popular constitui-se princípio do SUS, compreendendo as múltiplas ações que diferentes forças sociais desenvolvem, para influenciar a formulação, a execução, a fiscalização e a avaliação das políticas públicas e/ou de serviços básicos (saúde, educação, habitação, transporte, saneamento básico e outros), segundo Descritores em Ciências da Saúde (DECs).

No contexto internacional, tal conceito é descrito no Medical Subject Headings (MeSH), como somente o envolvimento dos membros da comunidade, em assuntos dessa comunidade, não apontando esse conceito como relacionado às políticas públicas de saúde. Tais diferenças conceituais prejudicam a busca por artigos científicos, nas bases de dados, por meio da combinação dos descritores 'Participação Popular' e 'Cuidados Paliativos', visto que, fundamentadas, nesta combinação, emergem diversas publicações não relacionadas ao conceito de Participação Popular disposto na legislação do SUS brasileiro. Dessa forma, optouse por um trabalho de reflexão teórica, que, após a busca exaustiva de experiências, nas bases de dados internacionais, identificaram-se algumas práticas semelhantes no contexto internacional, as quais possibilitaram subsídios, para a reflexão sobre uma possível política nacional, que contemple a Participação Popular no contexto dos CP.

Assim, preferiu-se artigos nacionais e internacionais que discorressem sobre a Participação Popular, no que tange à população de participar da formulação, planejamento, implementação ou avaliação de políticas públicas de saúde e serviços relacionados aos CP.

\section{RESULTADOS E DISCUSSÃO}

Em um contexto de envelhecimento populacional e de aumento do uso de tecnologias, para a manutenção da vida, torna-se cada vez mais urgente a necessidade de se buscar estratégias para que as preferências do paciente sejam respeitadas ${ }^{(10)}$. Busca-se propiciar um cuidado de qualidade, sistematizado e que atenda à grande parcela da população. Todavia, este objetivo somente torna-se tangível, ao se estabelecer a participação popular ao desenvolvimento e implantação de diretrizes voltadas para a prática clínica. Porém assegurar o envolvimento dos usuários apresenta-se como uma questão complexa, mesmo sendo defendida veementemente pelos gestores ${ }^{(11)}$.

A avaliação da participação de pacientes e familiares, em decisões relacionadas à morte e aos $\mathrm{CP}$, torna-se uma importante ferramenta, para a orientação e implementação de políticas públicas de saúde mais éticas ${ }^{(10)}$ e cabe aos gestores, envolvidos nos serviços de $\mathrm{CP}$, tentar desenvolver formas de incluir os pacientes e familiares nos diálogos sobre cuidados de fim de vida ${ }^{(7)}$.

Evidenciou-se, a partir de um estudo ${ }^{(12)}$ de revisão sistemática, com o objetivo de determinar o impacto da participação comunitária em saúde pública, aos cuidados de fim de vida, que serviços que envolvem a comunidade nas ações, voltadas à terminalidade nesses pacientes, possuem impacto positivo na formulação de políticas e pesquisas, cumprindo metas apontadas como complexas. Enfatiza-se, ainda, que é impraticável discutir assuntos relacionados ao fim de vida separados do contexto social, político e econômico das comunidades.

Tendo como base uma pesquisa ${ }^{(13)}$ qualitativa, realizada na Holanda, procurou-se compreender a influência e a participação dos pacientes e seus representantes na elaboração de políticas e tomadas de decisões em CP.

Neste país, a existência de redes em CP é uma realidade que visa assegurar a disponibilidade e variedade dos CP com qualidade e ajustar o serviço, constantemente, conforme a necessidade dos pacientes e familiares, por meio da participação popular. Acredita-se que o envolvimento popular proporcione ao paciente em CP voz ativa e a garantia dos princípios de autonomia e controle social durante seu tratamento.

$\mathrm{Na}$ análise dos dados do estudo citado, a participação social obteve expressividade na formulação e implementação das políticas, porém, durante os processos de avaliação, o envolvimento diminuiu e, na fase de ajuste dos serviços oferecidos, não houve influência satisfatória dos usuários. Percebe-se que a 
participação dos representantes da sociedade, nas fases de avaliação e ajustes, foi negligenciada pelos profissionais de saúde, pois, sendo os usuários leigos e membros da comunidade, subestima-se a sua capacidade de colaborarem com o planejamento das ações em saúde ${ }^{(13)}$.

Assim, demonstra-se que a participação popular de forma organizada, no contexto das políticas de saúde, é um direito do usuário e um feedback sobre a assistência prestada à população. No entanto verifica-se que há divergências entre os valores das ações comportamentais, resultantes de manifestações populares nas questões, envolvendo processos práticos e processos em longo prazo. Atitudes que requerem tomadas de decisões rápidas aparentam ter maior importância, em relação a processos mais burocráticos, tais como as políticas públicas de saúde ${ }^{(13)}$.

Assim, confirma-se, alicerçado em um estudo $^{(7)}$ europeu de revisão sistemática, realizado na Austrália, que há poucas informações que demonstrem o impacto da participação popular na saúde, principalmente, quando relacionadas diretamente aos $\mathrm{CP}$ e que o envolvimento da comunidade em CP ocorre, no âmbito de políticas específicas, tais como as políticas farmacêuticas.

Dessa forma, assinala-se que as experiências de países referentes à participação popular na formulação e gestão de políticas de saúde são escassas. Ao abordar o envolvimento social em $\mathrm{CP}$, os artigos direcionam-se às condições específicas, tais como qualidade dos serviços prestados ${ }^{(13)}$, disponibilização de fármacos $^{(7)}$ e terminalidade ${ }^{(12)}$.

Comparando-se os achados com a realidade brasileira, nota-se que a participação social na saúde do Brasil estruturou-se, na concepção do controle social, para que a comunidade civil colaborasse desde a criação de estratégias até a definição da alocação de recursos, a fim de que elas atendam aos interesses da coletividade. Logo, os principais locais, para a manifestação da participação e do controle social, para a efetivação das políticas de saúde, nas esferas do governo brasileiro, são os Conselhos e as Conferências de Saúde ${ }^{(14)}$.

Contudo, no contexto internacional, observa-se que não há, dentre as publicações exploradas, discussões sobre a organização referente à participação dos usuários em conselhos, conferências ou reuniões que estimulem e assegurem a relação estado- sociedade a práticas de vigilância e controle sobre as normas já existentes.

No Brasil, a publicação, a inserção dos $\mathrm{CP}$, em políticas públicas de saúde, é recente e, ainda, não há uma política específica para a normatização e organização desses cuidados no país. Faz-se necessária, portanto a utilização dos princípios doutrinários e organizativos do SUS, na implementação desses cuidados no país. Ao garantir o princípio da participação e controle social, torna-se democrático o processo de construção, implementação e gestão de políticas em saúde, além disso, volta-se a atenção para uma realidade que vai ao encontro das reais necessidades da comunidade em questão.

Os estudos que abordam terminalidade, por se tratarem de um assunto dilemático que exige uma avaliação interdisciplinar, aparecem como uma das temáticas que envolvem a população, nas tomadas de decisões e formulação de estratégias em CP, principalmente, no cerne das manifestações de vontades no fim de vida e suas implicações jurídicas e sociais.

Um exemplo que se aproxima dos Conselhos de saúde brasileiros é o do comitê consultivo de Maryland, nos Estados Unidos da América (EUA), voltado ao aconselhamento na formulação de políticas estatais para os CP, constituído por um grupo de especialistas interdisciplinares e defensores dos cidadãos. 0 Maryland State Advisory Council on Quality Care at the End of Life (MSAC) combina planejamento estratégico, engajamento público e reuniões programadas a fim de permitir comentários sobre propostas e políticas estatais.

O MSAC foi criado por uma legislação que o considera órgão permanente do governo de Maryland. O trabalho desenvolvido no MSAC, ao longo de oito anos, demonstrou que o modelo pode ser um catalisador eficaz para uma mudança positiva na formulação de políticas no final da vida ${ }^{(15)}$.

No Canadá, a Canadian Strategy for Cancer Control (CSCC), também, constitui-se um interessante mecanismo de participação popular, o qual começou, em janeiro de 1999 e envolveu mais de 130 profissionais de saúde e representantes da comunidade. Um aspecto crucial da implementação bem-sucedida da estratégia foi a participação precoce dos ministérios provinciais/ territoriais da saúde no processo de planejamento. Foram propostos diversos grupos de trabalho e duas conferências de partes interessadas, visando ao 
desenvolvimento de recomendações e estabelecimento de prioridades, para o controle do câncer no país. No relatório do conselho, ressaltou-se como prioridade a participação de pacientes, aos quais denominam 'sobreviventes', no desenvolvimento e implementação da CSCC, pois, para uma abordagem equilibrada ao controle do câncer, deve-se incluir a perspectiva e experiência do paciente. A voz dos pacientes e sobreviventes é trazida para a Mesa do Conselho pela Canadian Cancer Action Network (CCAN), uma coligação de grupos de apoio ao paciente/sobrevivente que representa uma ampla gama de tipos de câncer ${ }^{(16)}$.

Descreve-se, nas páginas iniciais da atual 'The Canadian Strategy for Cancer Control: 20172022', o depoimento de uma paciente conselheira da parceria 'National Survivorship Working Group'.Destaca-se que 700 canadenses pesquisadores, profissionais de saúde, acadêmicos, profissionais de saúde líderes do sistema e defensores dos pacientes - têm-se reunido, por intermédio da CSCC, a fim de tratar do crescente número de casos e mortes por câncer no país ${ }^{(17)}$.

$\mathrm{Na}$ Espanha, optou-se por um modelo de 'júri modificado do cidadão' organizado com a participação de 30 membros e quatro especialistas como testemunhas. Objetivou-se, com esse júri, avaliar a qualidade do tratamento do câncer, por meio de opiniões, experiências e percepções do paciente, buscando-se recomendações para melhorar o tratamento do câncer no país. Durante um dia, jurados representando 13 de 17 Comunidades Autônomas Espanholas foram atendidos, a fim de fazer recomendações, para melhorar a qualidade do tratamento do câncer. Foram identificadas preocupações quanto à fragmentação do cuidado, atrasos nos testes, duplicações e pouco apoio social e emocional.

Algumas estratégias, propostas pelo júri, incluíam um centro de atendimento 24 horas, continuidade nos CP e acompanhamento apropriado e apoio após o término da terapia. Destacou-se por esse estudo que, embora a realização do júri junto a um pequeno número de jurados possa parecer uma limitação, quando comparado com outras metodologias, o júri do cidadão é um mecanismo único de democracia pública que pode render ricos dados sobre as percepções e recomendações dos pacientes quanto a questões políticas, visto que organizações e lideranças de pacientes, na
Espanha, geralmente, não são convidados a participar do processo de tomada de decisão política ${ }^{(18)}$.

De um modo distinto, a participação popular é uma diretriz organizacional do SUS brasileiro, devendo-se, portanto prever estratégias, para que pacientes e seus familiares possam participar ativamente na construção, avaliação e melhorias das políticas públicas para os CP.

No Brasil, tem-se iniciado um movimento social informal, impulsionado por pacientes, visando à maior visibilidade das necessidades das pessoas que sofrem de doenças que limitam a vida. Recentemente, uma paciente em tratamento de câncer de mama metastático foi entrevistada, em um reconhecido programa de televisão brasileiro ${ }^{(19)}$, apresentando à comunidade o cotidiano de pacientes que vivenciam doenças fora de possibilidades de cura. Além disso, tal paciente tem impulsionado milhares de seguidores nas redes sociais e tem participado de eventos científicos, voltados aos CP no país, relatando as necessidades dos pacientes em diferentes contextos de prestação de serviço de saúde.

Assim, mediante a atual discussão de uma proposta de política pública específica, para os $\mathrm{CP}$ no país, devem-se buscar estratégias para que pacientes em CP e seus familiares participem ativamente da formulação de políticas de saúde nacionais, pois, ao abranger a opinião popular, garante-se que as ações em saúde sejam ofertadas de maneira assertiva às necessidades apresentadas pela comunidade. Destaca-se o papel da enfermagem, no fortalecimento desta participação, visto que esses profissionais encontram-se inseridos, em diferentes espaços de produção de cuidado, na gestão de políticas e serviços de saúde e em conselhos locais e municipais de saúde ${ }^{(20)}$.

Com esta reflexão, propõe-se que a organização dos serviços relacionados à prestação de $\mathrm{CP}$, no país, considere a participação de representantes de pacientes e familiares, nas instâncias colegiadas 'Conferências de Saúde e Conselho de Saúde', para que eles possam apresentar, de forma organizada, reivindicações mais específicas às suas necessidades até os órgãos competentes.

\section{CONCLUSÃO}

O presente estudo apresentou reflexões acerca da participação e controle social, no 
contexto político dos $\mathrm{CP}$, com importantes implicações para a efetivação deste princípio na organização dos serviços relacionados aos CP no SUS.

Observou-se pelas publicações encontradas, em bases de dados de periódicos e políticas de saúde de diferentes países, que poucos países contemplam, efetivamente, a participação e o controle social em suas políticas de saúde para os CP. Assim, poucos estudos contemplam o impacto dessa participação de países na qualidade dos serviços de CP.

Torna-se relevante o aprofundamento da discussão acerca da importância da participação e controle social voltados a pacientes em $\mathrm{CP}$ e seus familiares, no âmbito da formulação e gestão de políticas públicas, para esses cuidados no Brasil e do papel da enfermagem no fortalecimento desse direito.

Ressalta-se a necessidade de outros estudos que contemplem a temática, visando aprimorar as discussões e compartilhar experiências acerca da participação popular e dos impactos proporcionados por ela no âmbito dos CP.

\section{REFERÊNCIAS}

1- World Health Organization (WHO). Better palliative care for older people. Geneva: WHO; 2004.

2- Knaul FM, Agricultor PE, Krakauer EL, Lima L, Bhadelia A, Kwete XJ, et al. Alleviating the access abyss in palliative care and pain relief-an imperative of universal health coverage: The Lancet Commission report. Lancet 2018;391(10128):1391-1454. DOI: 10.1016/S0140-6736(17)32513-8

3- Vasconcelos BG, Pereira PM. Cuidados paliativos em atenção domiciliar: Uma revisão bibliográfica. Rev Adm Saúde 2018;18(70):1-18. DOI: https://dx.doi.org/10.23973/ras.70.85

4- Whitelaw S, Clark D. Palliative care and public health: An asymmetrical relationship? Palliat Care 2019;12:1-14. DOI: 10.1177/1178224218819745

5- Academia Nacional de Cuidados Paliativos (ANCP). Panorama dos cuidados paliativos no Brasil. São Paulo: ANCP; 2018.

6- Brasil. Resolução no 41, de 31 de outubro de 2018. Dispõe de diretrizes para a organização dos cuidados paliativos, à luz dos cuidados continuados integrados, no âmbito Sistema Único de Saúde (SUS). Diário Oficial da União 2018;1(225):276.
7- McConigley R, Shelby-James T, Currow DC. Promoting the consumer voice in palliative care: Exploring the possibility of using consumer impact statements. Health Expect. 2015;18(5):653-60. DOI: 10.1111/hex.12118

8- Brasil. Lei n. 8.142, de 28 de dezembro de 1990. Dispõe sobre a participação da comunidade na gestão do Sistema Único de Saúde - SUS e sobre as transferências intergovernamentais de recursos financeiros na área da saúde e dá outras providências. Diário Oficial da União 1990.

9- Mendes EC, Vasconcellos LDC. Cuidados paliativos no câncer e os princípios doutrinários do SUS. Saúde Debate 2015;39(106):881-92. DOI: 10.1590/0103-1104201510600030026

10- Belanger E. Shared decision-making in palliative care: Research priorities to align care with patients' values. Palliat Med. 2017;31(7):585-6. DOI: 10.1177/0269216317713864

11- Köpke S, Giordano A, Veronese S, Rahn AC, Kleiter I, Basedow-Rajwich B, et al. Patient and caregiver involvement in the formulation of guideline questions: Findings from the European Academy of Neurology guideline on palliative care of people with severe multiple sclerosis. Eur J Neurol. 2019;26(1): 41-50. DOI: 10.1111/ene.13760 12- Sallnow L, Heather R, Scott AM. The impact of a new public health approach to end-of-life care: A systematic review. Palliat Med. 2016;30(3):20011. DOI: $10.1177 / 0269216315599869$

13- Haarsma F, Moser A, Beckers M, Van Rijswijk $H$, Stoffers E, Beurskens A. The perceived impact of public involvement in palliative care in a provincial palliative care network in the Netherlands: A qualitative study. Health Expect. 2015;18(6):3186-200. DOI: 10.1111/hex.12308

14- Rolim LB, Cruz RSBLC, Sampaio KJAJ. Participação popular e o controle social como diretriz do SUS: Uma revisão narrativa. Saúde Debate 2013;37(96):139-47. DOI: 10.1590/S010311042013000100016

15- Rushton $\mathrm{CH}$, Schwartz J. A legislatively mandated council: A model for palliative care policy integration. Palliat Med. 2011;14(11):12405. DOI: $10.1089 / \mathrm{jpm} .2011 .0003$

16- Luciani S, Berman NJ. Status report. Canadian strategy for cancer control. Chronic Dis Can. 2000 [citado em 15 jan 2019]; 21(1):23-5. Disponível em:

https://www.ncbi.nlm.nih.gov/pubmed/10813690 17- Canadian Partnership Against Cancer. The Canadian Strategy for Cancer Control: 2017- 
2022. Toronto, On: Canadian Partnership Against Cancer; 2017.

18- Arrighi E, Blancafort S, Jovell AJ, Rubio MDN.

Quality of cancer care in Spain: Recommendations of a patients' jury. Eur J Cancer Care 2015;24(3):387-94. DOI: https://doi.org/10.1111/ecc.12208

19- Soares AM. Ana Michelle mostra seu cotidiano convivendo com câncer nas redes sociais [Entrevista a Conversa com Bial]. Rede Globo de Televisão 25 out. 2018. 1 vídeo (2 min). Disponível em: https://globoplay.globo.com/v/7115219 20- Ponte HMS, Silva AVJG, Pinto FRM, Aguiar FAR, Aviz ALM, Aires SF, et al. Ser enfermeiro, ser conselheiro: despertando para o controle social e saúde pública. Rev Bras Enferm 2019;72(1):134-9.

DOI: 10.1590/0034-7167-2017-0544

Nota: Financiamento pela FUNDECT e MS - PPSUS.

Recebido em: 01/06/2019

Aprovado em: 15/11/2019

Endereço de correspondência:

Jacqueline Resende Boaventura

Rua Marcelo Vitória 261, casa 14.

CEP: 79.622-042 - Três Lagoas/MS - Brasil

E- mail: jacque boaventura@yahoo.com.br 